\title{
Selbstbejahung und Selbstverneinung
}

\author{
Der Mut des Vertrauens und der Mut der Verzweiflung
}

〈Ich habe nichts mehr zu verlieren ...). So mag sich ein Mensch äußern, der seines Lebens müde geworden ist. Wenn wirklich nichts mehr zu verlieren ist, dann kann man sich auch selbst aufgeben, so der letzte müde Schluss, und ihm folgt vielleicht sogar der letzte Schuss, die letzte Fahrt, das letzte Wort in dieser Welt. - 〈Aber wir haben noch jemanden zu verlieren!) So auf der anderen Seite jene Menschen, die den Lebensmüden lieben und ihm einen guten Grund geben wollen, am Leben zu bleiben und seinen Weg noch nicht, ja überhaupt nie, gewaltsam zu beenden. Doch wie kann aus einem lebensmüden wieder ein lebensmunterer Mensch werden?

Nicht nur Suizidgedanken sind dem eigenen Leben eine Bedrohung; die Bedrohung lauert auch da, wo sie nicht einmal bemerkt wird: in einem Alltag, der scheinbar ganz normal verläuft, völlig unauffällig, aber so, dass der Mensch, der in ihm lebt, ein lebendig Toter ist. Dass das Leben lebensgefährlich ist, gilt somit nicht nur in Hinsicht auf die spektakulären Gefahren, die durch Überschwemmungen, Brände, Unfälle, Überfälle und die unzähligen anderen Weisen entstehen, auf die Menschen verletzlich in ihre Umwelt eingebunden sind und einander überdies das Leben schwer machen können, sondern auch in Hinsicht auf die Gefahren, die dem eigenen Leben durch einen selber drohen.

Dass am Ende alles gut wird, ist nicht gesagt. Gesagt sei lediglich, dass das lebendig Totsein, das Deprimiert-, Frustriert- und Verzweifeltsein zumindest auch etwas mit einem selbst zu tun hat. Die Selbstbezogenheit interessiert hier jedoch nicht, um irgendwelche Schuldzuweisungen daran anknüpfen zu können. Ganz im Gegenteil. Wohl wissend, dass der Zustand des Lebendig-Totseins auch auf tragische und traumatische Weisen eintreten kann und dass der Satz ¿Ich habe nichts mehr zu verlieren...) auch darauf verweisen kann, dass man in der Tat ohne eigene Schuld das verloren hat, was einem das Wichtigste im Leben war bzw. das, den- oder diejenige(n), wofür man da war - dies alles wohl wissend, sei hier lediglich gesagt, dass genau die Selbstbezogenheit des Unglücks oder Unglücklichseins, dies, dass es (mich selbst) trifft oder betrifft, eine Chance ist. 
Denn «mein` Umgang mit dem Unumgänglichen wird fortan bestimmen, wie das eigene Leben weitergeht, und wenn es kein Weiter geben sollte, dann zumindest, wie es endet. Sowohl ein würdiger Neubeginn wie ein würdiges Ende erfordert jedoch Mut - den Mut derVerzweiflung oder den Mut des Vertrauens oder alle beide, sofern der Mut desVertrauens ein Mut zum Leben ist, der nur dann bewahrt wird, wenn angesichts des Todes der Mut derVerzweiflung entsteht, der zwar stets verneint, aber gerade dadurch den Lebensmüden auch stärken kann, wenn das, was verneint wird, der erlebte Tod mitten im Leben ist. Einerseits scheinen Vertrauen und Verzweiflung einander entgegengesetzt zu sein, sofern der Mut des Vertrauens lebens- und selbstbejahend, der Mut der Verzweiflung jedoch selbst- und lebensverneinend ist; andererseits scheint die bedingungslose Bejahung des Lebens genau dadurch zustandezukommen, dass sie ihr Gegenteil, die Verneinung der jeweiligen Lebensbedingungen, in sich aufnehmen kann.

Wie aber kann diese dialektische Dynamik überhaupt in Gang kommen, wenn dem zu Tode betrübten Menschen doch vor allem Kraft fehlt, sowohl die Kraft zumVertrauen wie auch zumVerzweifeln? Wie kann 〈er selbst) noch weitergehen, wenn 〈es〉 nicht mehr weitergeht, wenn das Leben gleichsam zum Stillstand gekommen ist? Und wie könnte 〈er selbst〉 andererseits nicht mehr weitergehen wollen, wenn 'es〉 dennoch noch weitergehen sollte mit ihm? Sowohl das Leben hier und jetzt wie auch ein anderes Leben dort und dann, im Fall, dass der letzte〉 lebensmüde Schluss dennoch kein endgültiges Ende bringt, wäre nicht auszuhalten, wenn es nichts gäbe, was einen am oder im Leben halten würde. Wo aber gibt es einen letzten Halt?

Die Frage nach einem letzten (oder zumindest vorletzten) Halt spielt mit, wo von Vertrauen die Rede ist. Denn Vertrauen lässt sich nicht auf Selbstvertrauen reduzieren, sondern impliziert ein SichVerlassen-auf-einen-anderen. Wo man nicht wagt, sich zu verlassen, bleibt man bei sich - was jedoch nicht heißt, dass man sich, oder gar sich allein, vertrauen könnte. ${ }^{1}$ Umgekehrt bringt die Verzweiflung als Hoffnungslosigkeit (de-speratio) zum Ausdruck, dass die Hoffnung (spes) auf einen letzten Halt entweder schon aufgegeben wurde oder aber kämpfend kaum noch festgehalten werden kann. Im Folgenden werden einige Schlüsselszenen dieses Kampfes skizziert, und zwar mit Verweis auf Schriften von Menschen, die diesen Kampf um Leben oder Tod auf ihre je eigene Weise gekämpft haben und dessen allesentscheidenden Ernst ‘von innen heraus` kannten - unter ihnen

\footnotetext{
1 Vgl. hierzu meine Habilitationsschrift Vertrauen und Versuchung (Religion in Philosophy and Theology 51), Tübingen 2010, 11, 34f, 40, 62, 71, 85, 91, 109, 149, 248.
} 
Paul Tillich, Søren Kierkegaard, Friedrich Nietzsche, Martin Luther und Franz Rosenzweig.

\section{Der Mut der Verzweiflung und die Angst vor dem Dasein}

Die archetypische Ausgangssituation, die den Mut der Verzweiflung und/oder den Mut des Vertrauens hervorruft, ist Paul Tillich zufolge durch Angst charakterisiert. In seiner kleinen Schrift Der Mut zum Sein (1953) heißt es, in jeder Angst vor einer besonderen Situation sei die Angst vor der menschlichen Situation als solcher enthalten - die Angst, das eigene Sein zu verlieren. ${ }^{2}$ Diese generalisierte Grundangst kann laut Tillich nicht in Furcht vor etwas Bestimmtem verwandelt und aufgehoben werden. Die Angst als das Gewahrwerden möglichen Nichtseins und als Erfahrung der eigenen Endlichkeit gehört zur Existenz selbst. Tillich unterscheidet drei Typen der Angst, in denen das Nichtsein das Sein bedroht: die Angst vor Schicksal und Tod, in der die ontische Selbstbejahung des Menschen auf dem Spiel steht, die Angst vor Leere und Sinnlosigkeit, in welcher der Zweifel zur Verzweiflung mutiert, und die Angst vor Schuld und Verdammung, die mit der Verzweiflung darüber einhergeht, die eigene Bestimmung verfehlt zu haben. ${ }^{3}$

Diese verschiedenen Typen der Angst tragen alle zurVerzweiflung bei, doch treiben sie uns auch zum Mut als Alternative zurVerzweiflung: «Der Mut widersteht der Verzweiflung, indem er die Angst in sich hineinnimmt.» ${ }^{4}$ Dies ist der einzig wirksame Widerstand gegen die Verzweiflung, denn die existentielle Angst kann nicht beseitigt werden. Sie muss als zur conditio humana gehörig akzeptiert werden. Die Angst wird pathologisch, wenn das Selbst nicht fähig ist, seine Angst auf sich zu nehmen und wenn diese stattdessen zu einer Selbstbejahung treibt, die sich auf eine unrealistische, zwanghaft verteidigte Basis stützt, nämlich (in Bezug auf die Angst vor Schicksal und Tod) auf eine unrealistische Sicherheit, (in Bezug auf die Angst vor Schuld und Verdammung) auf eine unrealistische Vollkommenheit und (in Bezug auf die Angst vor Leere und Sinnlosigkeit) auf eine unrealistische Gewisheit. ${ }^{5}$ Daraus folgt, dass das Aufsichnehmen der Angst

2 P. Tillich, Der Mut zum Sein, Berlin/New York 1991 (unveränderter Nachdruck aus dem XI. Band der Ausgabe der Gesammelten Werke, erschienen 1969), 37.

3 Vgl. aaO., 38f, 44 und 47.

$4 \mathrm{AaO}, 56$

5 Vgl. aaO., 64. 
auch ein Aufsichnehmen von Unsicherheit, Unvollkommenheit und Ungewissheit mit sich bringt, sofern wir unseres Lebens nie sicher sind, von moralischer Perfektion immer entfernt bleiben und nach dem Sinn unseres Daseins permanent suchen müssen.

Wie wir die Angst auf uns zu nehmen haben, weil wir sie nicht beseitigen können, haben wir auch die Verzweiflung auf uns zu nehmen, und das können wir mit dem Mut der Verzweiflung. Tillich macht deutlich, dass der Begriff «Mut» ontologisch betrachtet werden muss, wenn er ethisch verstanden werden soll: 〈Mut〉 als menschlicher Akt und als Ausdruck einer Wertung ist ein ethischer Begriff, «Mut als Bejahung des Seins dagegen ist ein ontologischer Begriff. ${ }^{6}$ Im «Mut zum Sein kommen der ethische und der ontologische Aspekt zusammen. Tillich definiert den «Mut zum Sein` als den Akt, in dem der Mensch sein eigenes Sein bejaht trotz der Elemente in seiner Existenz, die im Widerspruch zu seiner Selbstbejahung stehen. Im englischen Wort mood und in Kombinationen wie Schwermut, Hochmut und Kleinmut kommt überdies zum Ausdruck, dass Mut auch eine Stimmung oder Seelenregung sein kann:

Mut ist eine Sache des Herzens, des Zentrums der Person; deshalb kann man für mutig auch beherzt sagen (das französische und englische Wort courage ist von coeur abgeleitet). [...] Mut ist Geistesstärke, die alles überwindet, was die Erlangung des höchsten Gutes bedroht. Zusammen mit Weisheit, Mäßigung und Gerechtigkeit gehört er zu den vier Kardinaltugenden. ${ }^{7}$

Wie sich der Mut als Tugend und Geistesstärke im sogenannten «Mut der Verzweiflung〉 zur Verzweiflung verhält, geht aus dem Zitat jedoch nicht hervor. Ist die Verzweiflung nicht eher eine Art Geistesschwäche und Untugend, in der zum Ausdruck kommt, dass man an den gegebenen Herausforderungen gescheitert ist und nun desperat, hoffnungs- und mutlos aufgibt? Tillich teilt diese Meinung über die Verzweiflung offenbar nicht, sonst könnte er sie nicht mit «Mut verbinden. Oder hat er es absichtlich auf eine paradoxale $\mathrm{Zu}-$ sammensetzung von Entgegengesetztem angelegt, um den Mut der Mutlosigkeit, die Tugend der Untugend und die Geistesstärke der Geistesschwäche hervorzuheben?

Wahrscheinlich liegt Tillich jedoch nicht so sehr am Paradoxalen als daran, den Ursprung einer bestimmten Form des Mutes zu bezeichnen - eines Mutes, der aus der Verzweiflung entsprungen und durch ihre Negativität hindurchgegangen ist, wodurch er sie auch

\footnotetext{
Vgl. aaO., 13f

$\mathrm{AaO}, 16$.
} 
überwunden hat. So bemerkt Tillich im Hinblick auf die Stoiker: «Seneca sagt, daß es keinen größeren Mut gebe als den aus der äußersten Verzweiflung geborenen. Aber kann der Stoiker als Stoiker - so muß man fragen - den Zustand der äußersten Verzweiflung erreichen?» ${ }^{8}$ Diese Frage ist berechtigt, ging es den Stoikern doch vor allem um die Ataraxia, die Seelenruhe ohne Sorgen, oder gar ohne Passionen, die vollkommene Apatheia. Die Verzweiflung dagegen zeichnet sich durch eine Leidenschaftlichkeit aus, die ohne inneren Kampf nicht zu beruhigen ist. Der Mut der Verzweiflung befasst den Mut in sich, diesen Kampf aufzunehmen.

\section{Formen verzweifelter Selbstverneinung}

Dass die 〈Verzweiflung〉 ein Begriff ist, der vielfältige Phänomene unter sich vereinen kann, zeigt Søren Kierkegaards klassische Phänomenologie der Verzweiflung in seinem Buch Sygdommen til Døden (1849). ${ }^{9}$ Im Ersten Abschnitt des Buches macht Kierkegaard zunächst in Unterabschnitt (A.) deutlich, dass die im Buchtitel genannte Krankheit zum Tode die Verzweiflung ist, widmet sich dann (B.) der Allgemeinheit dieser Krankheit und beschreibt schließlich (C.) die Gestalten dieser Krankheit, nämlich einerseits (in Hinsicht auf ein Ungleichgewicht in der menschlichen Synthesis, d.h. im Menschen als einem zusammengesetzten Wesen) ein Mangel an Endlichkeit oder Unendlichkeit, an Möglichkeit oder Notwendigkeit, und andererseits (in Hinsicht auf den Grad des Bewusstseins von der Krankheit) zum Einen die unbewusste Verzweiflung dessen, der sich nicht darüber im Klaren ist, dass er ein ewiges Selbst hat bzw. verliert, und zum anderen die Verzweiflung dessen, der sich zwar seiner selbst bewusst ist, aber entweder verzweifelt nicht er selbst sein will (in der Verzweiflung der Schwachheit) oder verzweifelt er selbst sein will (in der Verzweiflung des Trotzes). Da es dem Verzweifelten weder glückt, nicht er selbst zu sein, noch, er selbst zu sein, sind die beiden letztgenannten Formen derVerzweiflung aufeinander rückführbar.

Unterabschnitt (A.) gliedert sich wiederum in drei Abschnitte, in deren letztem (A.C.) erklärt wird, dass Verzweiflung zwar mit dem leiblichen Tode enden kann, dass deren Qual aber gerade darin besteht, dass man nicht sterben kann. Obwohl der Verzweifelte in sei-

$8 \mathrm{AaO}, 23$

9 Ich halte mich hier an folgende Übersetzung: S. Kierkegaard, Die Krankheit zum Tode. Der Hohepriester - der Zöllner - die Sünderin, in: ders., Gesammelte Werke, 24. und 25. Abt., hg. u. übers. von E. Hirsch und H. Gerdes, Gütersloh ${ }^{4} 1992$ (= KT). 
ner Hoffnungslosigkeit nicht sterben kann, leidet er dennoch unter einer Art «ohnmächtige[r] Selbstverzehrung, die nicht vermag was sie selber will $»^{10}$. Zum Verzweifeln ist einerseits, dass der verzweifelte Mensch sich nicht loswerden kann, und andererseits, dass er es nicht aushalten kann, er selbst zu sein. ${ }^{11}$ In dieser paradoxen Doppeltheit von selbstverzehrendem Selbstverhaftetsein ist derVerzweifelnde sich selbst eine Plage geworden. In seiner Verzweiflung verneint er sich selbst - doch vergeblich. Seine Selbstverneinung führt gerade nicht zur Auslöschung seiner selbst, aber auch nicht zur Selbstbejahung. Er will sich selbst und Gott, der ihn ins Leben rief, loswerden, ohne dass er dies könnte:

Das Selbst, das er verzweifelt sein will, ist ein Selbst das er nicht ist (denn das Selbst sein wollen, das er in Wahrheit ist, ist ja das gerade Gegenteil von Verzweiflung), er will nämlich sein Selbst losreißen von der Macht, die es gesetzt hat. Indes das vermag er trotz allem Verzweifeln nicht; trotz aller Anstrengung der Verzweiflung ist jene Macht die stärkere, und zwingt ihn das Selbst zu sein, das er nicht sein will. ${ }^{12}$

Im Unterschied zu Tillich nimmt Kierkegaard an, dass sich im Menschen «etwas Ewiges» ${ }^{13}$ findet, so dass die Verzweiflung sein Selbst einerseits nicht ganz verzehren kann, der Tod ihn andererseits aber auch nicht von dieser Krankheit befreien kann. Dies wird dort übersehen, wo jemand versucht, sich durch Selbstmord loszuwerden. Sich umzubringen ist kein Ausweg.

Kierkegaard macht einen anderen Vorschlag:

«Möglichkeit ist das Eine, das rettet. Wenn einer ohnmächtig wird, ruft man nach Wasser, Eau de Cologne, Hoffmannstropfen; wenn aber einer verzweifeln will, so heißt es: schaff Möglichkeit, schaff Möglichkeit, Möglichkeit ist das Einzige, was rettet; [...] denn ohne Möglichkeit kann ein Mensch gleichsam keine Luft bekommen.» ${ }^{14}$

Dass in einer scheinbar ausweglosen Situation neuer Lebensmut mobilisiert werden kann, setzt voraus, dass diese Situation anders gesehen wird, und zwar unter dem Gesichtspunkt dessen, wie aus der Enge wieder Weite wird, unter dem Gesichtspunkt dessen, wie

\footnotetext{
${ }^{10} \mathrm{KT} 14$.

11 Vgl. KT 15

12 KT 16.

13 KT 17.

${ }^{14} \mathrm{KT} 35 f$
} 
die Lage zum Guten gewendet werden kann, so dass man wieder befreit aufatmen kann. Die Möglichkeit als Gegengift gegen die Verzweiflung ist insbesondere dem Glaubenden zu eigen, der es ganz Gott anheimstellt, wie ihm geholfen werden soll, aber der glaubt, dass alles möglich ist bei Gott, alles in jedem Augenblick. ${ }^{15}$

Christiane Tietz hat sowohl im Anschluss an Kierkegaard wie an Tillich einen christlichen Begriff von Selbstannahme entfaltet mit der rechtfertigungstheologischen These, dass Freiheit $z u$ sich selbst in der Freiheit von sich selbst gründet. ${ }^{16}$ Selbstbejahung sei dem Menschen nicht direkt möglich; sie sei nur möglich durch die Rechtfertigung, in der der Mensch als Person von seinen Schwierigkeiten und Vorzügen unterschieden wird. ${ }^{17}$ Strukturell lasse sich die christliche Selbstannahme als mehrfache Doppelbewegung beschreiben: Der Mensch müsse seine Abhängigkeit von der Rechtfertigung bejahen (Bewegung hin zu sich), um sich dann in der Rechtfertigung von seinen Schwierigkeiten und Vorzügen unterscheiden zu lassen (Bewegung weg von sich). Nur durch diese Unterscheidung könne er diese als die seinen bejahen (Bewegung hin zu sich), und nur dadurch sei eine Veränderung der Schwierigkeiten möglich (Bewegung seiner selbst).

Der Vorteil dieser Beschreibung von Selbstbejahung ist die Betonung der Prozessualität: die eigenen endlichen Beschaffenheiten werden nicht sanktioniert, sondern als veränderliche respektiert. Freilich stellen sich auch Fragen: Ist es wirklich unmöglich, sich selbst unter Absehung von der Rechtfertigungslehre zu bejahen? Nietzsche hat diese Position durch die Entwicklung einer lebensphilosophischen Alternative herausgefordert. Dazu später mehr. Einmal angenommen, man folgt der rechtfertigungstheologischen Linie - ist die Bewegung darin lediglich ein Zu-sich-hin und Weg-von-sich, oder wird dieses Oszillieren nicht verkompliziert durch den Christus extra nos und den Christus in nobis, durch den die Bewegung orientiert und auf sich hin umgelenkt wird? Und lösen sich die Probleme in der Selbstannahme allein dadurch, dass zwischen Person und Werk differenziert wird, oder wäre nicht vielmehr eine Selbstannahme vonnöten, die Person und Werk zusammenhalten kann?

Die Schwierigkeiten und Vorzüge einer Person zeigen sich zuallererst in der Art, wie sie sich zu sich selbst und zu anderen verhält. Soll

15 Vgl. KT 37.

${ }^{16} \mathrm{Vgl}$. Ch. Tietz, Freiheit zu sich selbst. Entfaltung eines christlichen Begriffs von Selbstannahme (Forschungen zur systematischen und ökumenischen Theologie 111), Göttingen 2005,Vorwort.

${ }^{17}$ Vgl. aaO., 208. 
sich die Person selbst mit allen Schwächen und Stärken annehmen, muss ihr Sich-Verhalten ihr selbst individuell zurechenbar sein. Den Gedanken, dass die Selbstannahme nichtsdestotrotz nur indirekt möglich ist, nämlich über den Umweg des Angenommenseins durch Gott und dem gläubigen Sich-Annehmen als derart angenommen, entfaltet Tietz unter Berücksichtigung von Tillichs Vorschlag, Rechtfertigung als Gottes Annahme des Unannehmbaren zu verstehen. ${ }^{18}$ Dass die Annahme durch Gott voraussetzungslos ist, macht es dem Menschen möglich, seine eigene Existenz zu bejahen. Den Mut zum Sein kann er demnach nicht aus sich selbst heraus hervorbringen. Vielmehr entspringt dieser allein daraus, dass sich der Mensch von Gott angenommen fühlt. Die totale Bejahung des Menschen in allen Aspekten seines Daseins wird jedoch sofort wieder zurückgenommen, wenn Tillich mit Oswald Bayer dafür kritisiert wird, er übersehe die Konkretheit und Personalität des Rechtfertigungsgeschehens; es müsse dem Menschen gesagt werden, worin, weshalb und woraufhin er angenommen ist. ${ }^{19}$ Die durch die Rechtfertigungslehre vermittelte Selbstbejahung ist demzufolge eine differenzierte.

An dieser Stelle mag einem Kierkegaards Gedanke in den Sinn kommen, dass man auch so verzweifeln kann, dass man von Reue und Gnade nichts hören will, sondern auch über seine Sünde geradezu verzweifeln will mit den Worten: (ich vergebe mir das niemals ${ }^{20}$ - obwohl Gott doch dieVergebung im umfassendsten Sinne zugesagt hat, d.h. sowohl Vergebung schuldhafter Taten wie auch sündhaften, selbst- und gottwidrigen Seins. Gegen Kierkegaards Definition des Glaubens als Zustand, in dem schlechterdings keine Verzweiflung ist - «indem es sich zu sich selbst verhält, und indem es es selbst sein will, gründet sich das Selbst durchsichtig in der Macht, welche es gesetzt hat» - wird allerdings geltend gemacht, dass es eine Unterbestimmung des Glaubens sei, wenn man ihn nur als impliziten Gottesbezug (indem der Mensch sich auf sich selbst bezieht, bezieht er sich auf Gott) versteht. ${ }^{21}$ Dagegen wird eingewandt, Glaube sei wesentlich expliziter Gottesbezug. Dieser Einwand verwundert ein wenig angesichts der oben angeführten Texte, aus denen hervorgeht, dass mit dem Glauben an Gott bei Kierkegaard auch bestimmte propositionale Glaubensinhalte verbunden sind, z.B. dass bei Gott allezeit alles möglich ist.

\footnotetext{
${ }_{18} \mathrm{Vgl} . \mathrm{aaO} ., \$ 2,16 \mathrm{ff}$.

19 AaO., 22, mit Verweis auf O. Bayer, Theologie (Handbuch systematischer Theologie 1), Gütersloh 1994, 272

${ }^{20} \mathrm{Vgl}$. KT 112

${ }^{21}$ Vgl. Tietz 2005, 211, mit Verweis auf KT 134.
} 
Zudem erfasst der indirekte wie der explizite Bezug auf Gott ja nur das am Glauben, was Menschenwerk ist, während das rechtfertigungstheologisch Entscheidende nicht das opus hominis, sondern das opus dei sein dürfte: Gottes gnädiges Sich-Beziehen und -Bezogenhaben auf den Menschen, ganz unabhängig davon, wann und wie der Mensch auf diese ihn begleitende und ihm vorausgegangene Gnade reagiert. Nur wenn der Glaube auch als das verstanden wird, was Gott am und im Menschen wirkt, kann dem menschlichen Festhaltenwollen an der Verzweiflung (und damit an der Selbstverneinung) wirksam der Boden entzogen werden. Denn dann kann der verzweiflungsvolle Mensch sich dessen gewiss sein, dass er in Gott eine Kraftquelle gegen die Verzweiflung findet, und dann kann auch das eigene «Nicht-mehr-glauben-Können〉 (oder eher: Nicht-mehr-glauben-Wollen) keine Ausrede mehr liefern.

Tietz argumentiert, dieVerzweiflung sei dann ausgetilgt, wenn man «durch sein Selbstsein hindurch immer auch seinen Grund, eben Gott, wahrnehmen kann.»22 Dem ist hinzuzufugen, dass es darauf ankommt, wie man Gott wahrnimmt, denn wenn Gott zwar wahrgenommen wird, der christliche Glaube an Gott aber sehenden Auges und ausdrücklich (modo ponendo) für Unwahrheit erklärt wird, dann wird sowohl an derVerneinung Gottes wie an der Selbstverneinung und damit an derVerzweiflung festgehalten. ${ }^{23}$ Deshalb unterstreicht Kierkegaard, dass das Selbst allein dann gesund und von Verzweiflung frei ist, wenn es eben dadurch, dass es verzweifelt hat, d.h. durch die Verzweiflung hindurch- und über sie hinweggekommen ist, in Gott gründet, und zwar sich selbst durchsichtig. ${ }^{24}$ Dass der Mensch im Glauben sich selbst durchsichtig sei und vollständiges Bewusstsein

${ }^{22}$ Vgl. Tietz 2005, 103

${ }^{23}$ Vgl. KT 126-134. Freilich lässt sich fragen, ob man in diesem Fall wirklich Gott wahrgenommen hat. Wenn Gott Liebe ist, wird er niemanden zwingen, ihn als Gott anzuerkennen. Die Möglichkeit, Gott sehenden Auges abzulehnen, setzt allerdings voraus, dass man die eigene Sicht gleichsam verschleiern und Gott zu etwas machen kann, was er nicht ist: zu einem Abgottkandidaten. Man sieht und sieht doch nicht.

${ }^{24}$ Vgl. KT 26. Im dänischen Text heißt es: «thi kun da er Selvet sundt og frit fra Fortvivlelse, naar det, just ved at have fortvivlet, sig selv gjennemsigtigt grunder i Gud» (SKS 11, 146). Die deutsche Übersetzung gibt das Verb wieder als ein Sich-Gründen, doch wenn dies der Fall wäre, stünde im Dänischen nicht nur "grunder», sondern «grunder sig». Dieselbe Fehlübersetzung findet sich beim ersten und letzten Auftreten jener Formel, die den Zustand des Selbsts beschreibt, wenn die Verzweiflung ganz und gar ausgetilgt ist: «indem es sich zu sich selbst verhält, und indem es es selbst sein will, gründet sich das Selbst durchsichtig in der Macht, welche es gesetzt hat [grunder Selvet gjennemsigtigt i den Magt, som satte det]» (KT 10 und 134; SKS 11, 130 und 242). Die Abkürzung SKS 11 verweist auf Søren Kierkegaards Skrifter, Bd. 11, hg. von Søren Kierkegaard Forskningscenteret, Kopenhagen 2006. Nur in der zuerst zitierten 
davon habe, wie er beschaffen ist ${ }^{25}$, sagt Kierkegaard damit jedoch nicht. Im Dänischen ist sig selv gjennemsigtigt nicht adjektivisch, sondern eine zum Verb 'gründen` gehörende adverbiale Bestimmung, und an anderen Stellen ist nur von durchsichtigem, nicht von sich selbst durchsichtigem Gründen die Rede. Tietz erklärt das durchsichtige Gründen in Gott anhand der schönen Analogie zu einem klaren Gebirgssee: wie man durch ihn hindurch auf seinen Grund sehen kann, könne man durch das Selbst hindurch auf dessen Grund, d.h. auf Gott, sehen. ${ }^{26}$ Ist der Mensch für Gottes heilsames Wirken transparent geworden, ist er auf dem Weg dazu, von der Krankheit zum Tode zu gesunden.

Der Glaube bringt ihn auf diesen Weg. Doch beinhaltet auch der Glaube einen Kampf des Glaubenden mit sich selbst. Inwiefern die Selbstbejahung im Glauben in gewisser Weise auch eine bestimmte Form von verzweifelter Selbstverneinung in sich einschließt, ist im Folgenden noch näher auszuführen. $\mathrm{Zu}$ erhellen ist zudem, weshalb Selbstannahme ausgerechnet durch den Selbstverlust hindurch geschieht.

\section{Der Mut, sich selbst zu verlieren, um sich wie- der zu gewinnen}

Kierkegaard erklärt, dass sich keine Form der Verzweiflung undialektisch bestimmen lässt, sondern allein, indem man auf das ihr Entgegengesetzte reflektiert. ${ }^{27}$ Dies bedeutet, dass der Glaube als verzweiflungsfreier Zustand mit in den Blick kommen muss, wenn die Verzweiflung recht verstanden sein will. Nun ist der Glaube jedoch nicht nur gegen die Verzweiflung gerichtet. Die Verzweiflung kann auch «der Durchgang zum Glauben" ${ }^{28}$ sein. Die zum Glauben hinführende Verzweiflung ist allerdings eine besondere Form der Verzweiflung: Verzweiflung vermöge des Ewigen, mit dessen Hilfe das Selbst «den Mut» hat, «sich selbst zu verlieren, um sich selbst zu gewinnen» - wohingegen das Selbst in der trotzigen Form der Verzweiflung gar nicht erst damit anheben will, sich selbst zu ver-

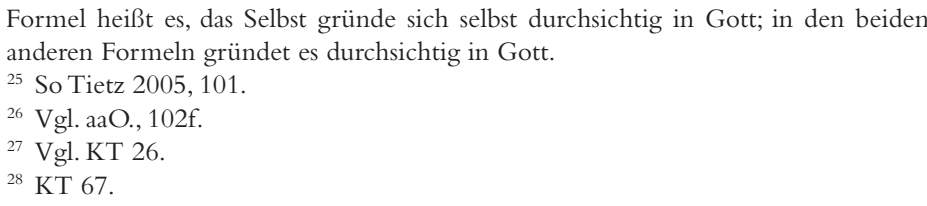


lieren, sondern lediglich es selbst sein will. ${ }^{29}$ Im Trotz will das Selbst über es selbst verfügen oder gar sich selbst erschaffen, d.h. sein Selbst zu dem Selbst machen, das es sein will. Es will sein eigener Herr sein und duldet keinen Herren über sich. Es will auch keine Hilfe von einem anderen als allein sich selbst, obwohl es sich selbst gerade nicht helfen kann, denn es will sich nicht verlieren und kann sich deshalb auch nicht gewinnen bzw. wiederfinden.

Kierkegaard kann die Verzweiflung im Zweiten Abschnitt des Buches auch als Sünde explizieren. Die Verzweiflung über die Sünde der Verzweiflung ist potenzierte Sünde. Doch ist diese ebenfalls dialektisch aufzufassen. Sie kann entweder zum Glauben hin- oder von ihm wegführen:

$\mathrm{Da}$ das Dialektische da ist [...], darf niemals vergessen werden, es ist ja damit gegeben, daßVerzweiflung auch das erste Moment im Glauben ist. Geht die Richtung hingegen vom Glauben fort, vom Gottesverhältnis fort, so ist Verzweiflung über die Sünde die neue Sünde. [...] Ärgernis ist ja dergestalt als aufgehobene Möglichkeit ein Moment im Glauben; jedoch Ärgernis, mit der Richtung vom Glauben fort, ist Sünde. ${ }^{30}$

Das Bewusstsein von der eigenen Verzweiflung und damit von der eigenen Sünde ist die Voraussetzung dafür, glaubend die Sündenvergebung zu suchen. Wer dagegen Ärgernis nimmt, d.h. an der Vergebung der Sünden verzweifelt, will nicht glauben und hält dadurch an der negativen Möglichkeit fest, die ansonsten im Glauben aufgehoben wird. Die Verzweiflung als erstes Moment im Glauben wird durch den Glauben überwunden, und zwar indem das verzweifelte Selbst den Mut bekommt, sich zu verlieren.

Wie Arne Grøn herausstellt, verweist die Schlüsselformulierung 'sich selbst verlieren〉 (at tabe sig selv) auf etwas, das man selbst tut. ${ }^{31}$ Auch das verzweifelte Sich-nicht-verlieren-Wollen ist etwas, das man selbst tut. Grøns gründliche und scharfsinnige Analyse zeigt überdies, dass den beiden einander dialektisch entgegengesetzten Formen von Verzweiflung auch zwei einander entgegengesetzte Formen von Selbstverlust entsprechen. ${ }^{32}$ Die größte Gefahr ist, sich

\footnotetext{
29 Ebd.

${ }^{30}$ KT 117 f Anm.

31 A. Grøn, Subjektivitet og negativitet: Kierkegaard, Kopenhagen 1997, 159f. Dieses Buch gehört zum Besten, was über Kierkegaard und die Thematik des Selbstseins und -werdens geschrieben wurde, und allein schon um dies verstehen zu können, lohnt es sich, die dänische Sprache zu erlernen.

${ }^{32}$ Im Folgenden stütze ich mich auf Grøn 1997, 168f, 376-382.
} 
selbst dauerhaft zu verlieren durch eine Form von Verzweiflung, die das Selbst verschließt und es für sich und andere unzugänglich macht. Andererseits kommt das Selbst nicht darum herum, sich zu verlieren. Es muss sich verlieren, um sich wiedergewinnen zu können, und zwar durch eine Form von Verzweiflung, die es offenhält und in einen Kampf mit sich selbst hineinführt.

1.) Die zu vermeidende Form von Verzweiflung ist die unbemerkte Verzweiflung, gegen die man nicht kämpft, weil man sich bereits aufgegeben hat. Der Selbstverlust hierbei ist schleichend und geschieht durch selbstvergessenes Dahinleben, ohne dass es einem aufgeht, dass Selbstsein eine Aufgabe enthält. Diese unbemerkte Verzweiflung, die sich nur indirekt z.B. in Ausweichmanövern und Gleichgültigkeit äußert, ist «das wahrhaft Entsetzliche»" ${ }^{33}$. Hier gibt es keine Hoffnung mehr, denn man ist sich abhanden gekommen, ohne dass einem dies bewusst geworden wäre. Dabei leidet man unter einer tödlichen Krankheit und bringt doch selbst den Tod mitten im Leben hervor - dadurch, dass man sich selbst um das Leben und um die eigene Lebensaufgabe betrügt und Schaden an der Seele nimmt. Ein Mensch, der sich aufgegeben hat, kann jener Lebensaufgabe nicht mehr nachkommen, die darin besteht, dass er er selbst wird und andere liebt. ${ }^{34}$ Dieser fatale Selbstverlust schließt aus, dass man sich je wiedergewinnen kann, weil man ungerührt mit ihm lebt und gar nicht mehr durch ihn bewegt wird. Für den, der sich selbst aufgegeben hat, verliert das Leben seinen Sinn.

2.) Die zum Sich-Wiedergewinnen hinführende Verzweiflung ist die bewusste Verzweiflung, die man bekämpft, weil man sich noch nicht aufgegeben hat. Deshalb kann man dem Widerwillen wider sich entgegenwirken und versuchen, ein neues Selbst-Verständnis zu erringen. Der damit zusammenhängende Selbstverlust ist die Einsicht, dass man sich verloren hat. Man sieht ein, dass man ein anderer ist, als man sich vorgestellt hat. Nur wer sich dessen bewusst ist, dass er krank ist, kann geheilt werden und sich wiederfinden. Wer nicht einmal auf die Gefahr aufmerksam wird, verliert sich so, dass er für sich selbst verloren ist. Wer die Gefahr bemerkt, muss durch dieVerzweiflung hindurch: «das Selbst muß gebrochen werden, um Selbst zu werden» (KT 65). Das Selbst wird gebrochen in dem Sinne, dass sein Bewusstsein, sein eigener Herr zu sein, gebrochen wird. Es soll sich unter sich selbst demütigen und einräumen, dass

\footnotetext{
${ }^{33} \mathrm{KT} 30$.

${ }^{34}$ Letztgenannte Lebensaufgabe wird von Kierkegaard näher beschrieben in jener Schrift, die das komplementäre Gegenstück zur Krankheit zum Tode ist: Der Liebe Tun (Kjerlighedens Gjerninger) von 1847.
} 
das, wozu es nicht stehen will, auch zu ihm selbst dazugehört. Es ist und wird nur dadurch es selbst, dass es sich auch durch seine negativen Möglichkeiten hindurch verstehen lernt - durch die zahllosen Möglichkeiten, nicht es selbst zu sein. Wenn es diese Möglichkeiten an sich selbst entdeckt, verzweifelt es an sich, weil es bemerkt, dass es «das Ewige und sich selbst verloren $»^{35}$ hat. Doch dieser Selbstverlust und die Verzweiflung daran ist notwendig, denn nur so entsteht der Mut, durch die Verzweiflung hindurchzugehen, um zu sich zurückzukommen und sich wiederzufinden, nachdem man sich verloren hat.

Man gewinnt sich folglich nicht zufällig, sondern durch den Verlust seiner selbst. Wie schon zitiert, heißt es bei Kierkegaard: "vermöge des Ewigen hat das Selbst den Mut, sich selbst zu verlieren, um sich selbst zu gewinnen ${ }^{36}$. Diesen Mut braucht das Selbst, denn seine Selbstwerdung geschieht gerade durch das Einholen desVerlustes, und dies wiederum bedeutet: durch die Überwindung der Verzweiflung. Anders als die mit Hoffnungslosigkeit einhergehende Verzweiflung ist der Mut eine hoffnungsvolle Weise, sich zur Zukunft zu verhalten. Wer Mut hat, nimmt das auf ihn Zukommende an. Formen des Mutes (Dänisch: mod), sind z.B. Geduld (tålmod) und Freimut (frimodighed). Diese Formen des Mutes sind erforderlich für den Kampf, den das Selbst mit sich selbst und mit seinem eigenen Umgang mit seiner Lebenszeit führen muss. Im Selbst ist ein Widerstand gegen sich selbst, ein Kampf im Willen, der darum geht, dass es nicht zu sich selbst und seiner eigenen Geschichte stehen will. Der Kampf erfordert den Mut, sich der Negativität zu stellen, in der man sich bereits befindet.

Denn nur dann, wenn man dieser Negativität begegnet und sie als (bereits verwirklichte oder verwirklichbare) Möglichkeit seiner selbst annimmt, kann das verneinte Selbst losgelassen, die Selbstverneinung überwunden und das durch die Verneinung Verlorene verändert wiedergewonnen werden.

\section{Selbstbejahung als Selbstüberwindung}

Inwiefern Selbstwerdung nicht nur Selbstverwandlung durch überwundene Selbstverneinung mit sich führt, sondern die Selbstbejahung ihrerseits Selbstverneinung in sich befasst und deshalb Selbstüberwindung bleibt, lässt sich auch von Friedrich Nietzsche lernen. Nietzsche und Kierkegaard kommen darin überein, dass Selbstbejahung der Negativität zum Trotz geschieht.

${ }^{35} \mathrm{KT} 61$

${ }^{36} \mathrm{KT} 67$. 
Tillich macht darauf aufmerksam, dass bei Nietzsche der Mut die Macht des Lebens ist, sich trotz der Zweideutigkeit des Lebens zu bejahen, während die Negation des Lebens als Ausdruck der Feigheit von ihm verworfen wird. ${ }^{37}$ Die Selbstbejahung des Lebens schließt Selbstbewahrung und Wachstum ein. Im Leben als Prozess aktualisiert sich die Seinsmacht, und indem sie sich aktualisiert, überwindet sie das im Leben, was das Leben negiert, obwohl es zum Leben gehört. Zum Leben gehört auch Leiden, Altwerden und Sterbenmüssen. Dementsprechend gehört zur Selbstbejahung die Bejahung des Lebens wie auch die Bejahung der zum Leben gehörenden Lebensbedrohung. Tillich verweist auf das Kapitel «Von den Predigern des Todes» in Also sprach Zarathustra (1883-1885), wo es über die «Schwindsüchtigen der Seele», die selber gerne tot sein wollen, heißt: «Ihnen begegnet ein Kranker oder ein Greis oder ein Leichnam; und gleich sagen sie «das Leben ist widerlegt!) Aber nur sie sind widerlegt und ihr Auge, welches nur das eine Gesicht sieht am Dasein.» ${ }^{38}$ Wer nur dieses eine Gesicht sieht, übersieht die anderen, lebensfrohen Gesichter des Lebens.

Nietzsche alias Zarathustra karikiert und kritisiert u.a. die Einstellung, man solle besser keine Kinder zeugen, weil man doch nur Unglückliche gebiert. Doch richtet sich sein beißender Spott auch gegen diejenigen, "denen das Leben wilde Arbeit und Unruhe ist», denen «das Schnelle, Neue, Fremde» lieb ist; diese Menschen fragt er, ob sie nicht des Lebens müde seien und sich selbst schlecht ertragen, und er hält ihnen vor: «euer Fleiß ist Fluch und Wille, sich selber zu vergessen. Wenn ihr mehr an das Leben glaubtet, würdet ihr weniger euch dem Augenblicke hinwerfen. Aber ihr habt zum Warten nicht Inhalt genug in euch - und selbst zur Faulheit nicht!» ${ }^{39}$ Selbstbejahung beinhaltet demnach auch den Mut, sich selbst auszuhalten und nicht vor sich und den eigenen Problemen zu fliehen. Eine gleichermaßen abgelehnte Flucht ist die Flucht vom Diesseits ins Jenseits, die Vertagung des jetzigen auf ein «ewiges〉 Leben. Ob der Tod oder das ewige Leben gepredigt wird, das gilt Zarathustra gleich: «wofern sie nur schnell dahinfahren!» ${ }^{40}$ Während sich der Ernst des Lebens für Kierkegaard gerade darin zeigt, dass es individuell erlebt wird von Menschen, die jede(r) für ihr ewiges Selbst Sorge zu tragen haben, das sie zwar nie loswerden, aber gleichwohl vergessen und

\footnotetext{
37 Vgl. Tillich 1991, 30 .

${ }^{38}$ F. Nietzsche, Also sprach Zarathustra, in: Werke in drei Bänden, Bd. 2, hg. v. K. Schlechta, Darmstadt 1997, 275-561, hier 310 (Erster Teil).

${ }^{39} \mathrm{AaO}, 311$.

40 Ebd.
} 
sozusagen 〈totleben〉 können, zeigt sich der Ernst des Lebens für Nietzsches Zarathustra in der Kürze des von den Einzelnen gelebten Lebens, das gerade als ambivalentes und vielgesichtiges einmalig und unwiederbringlich ist. Dieses Leben wird bejaht, indem es bis zu seinem Ende in vollen Zügen gelebt wird.

Das eigene Leben wird bejaht, indem es so gelebt wird, dass das eigene Selbst ganz «mit dabei ist. Nicht Selbstverleugnung, sondern Selbstbestätigung ist Zarathustras Losung. Im Kapitel ‘Von den Tugendhaften heißt es poetisch: «Es ist euer liebstes Selbst, eure Tugend. Des Ringes Durst ist in euch; sich selber wieder zu erreichen, dazu ringt und dreht sich jeder Ring." ${ }^{41}$ Das liebste Selbst ist jene Dimension des Selbstseins, die am einfachsten anzunehmen ist: das eigene Goethe'sche Edel-, Hilfreich- und Gutsein und das Schiller'sche Handeln nach dem Maßstab des Ideals. Auch dies, dass man unterwegs womöglich Eigenschaften an sich kennenlernt, die sehr schwer anzunehmen sind, und dass es sich womöglich herausstellt, dass man in einer entscheidenden Situationen versagt hat, muss dennoch wieder dorthin zurückführen, wovon man ausgegangen ist. Dass man sich selbst unterwegs verfehlt, ist genauso menschlich wie es menschlich ist, sich wieder erreichen zu wollen, um es mit sich selbst wieder aufnehmen zu können. An der Stelle jedoch, wo der 〈Ring〉 aus der Bahn läuft oder ohne Rundung unabgerundet abbricht, trifft die Metapher des Ringes auf das Menschsein nicht mehr zu. Ein Ring ist per definitionem ein geschlossener Kreis, während ein Menschenleben ein unabgeschlossener Gang ist, der mitunter danebengeht.

Tillich liest aus dem Wort vom Ring folgenden Sinn heraus, den die Selbstbejahung in der Lebensphilosophie habe: «Das Selbst hat sich, aber zugleich versucht es, sich zu erreichen.» ${ }^{42}$ Die Frage ist, ob es sich wirklich hat. Man könnte sagen, dass es sich hat, indem es und solange es ist. Sofern es sich jedoch zugleich zu erreichen versucht, hat es sich gerade nicht. Es ist nicht gesagt, dass es sich in der Tat erreicht. Das eigene Leben ist kein fertiger Ring, sondern ein fortgesetztes Ringen. Es ist ein Ringen mit sich selbst um sich selbst. Sofern man sich verfehlt, ist das eigene Leben nicht länger rund und schön, sondern schief und am Ziel vorbei gegangen. Die Tugend kann aus diesem Grund nicht in etwas bestehen, an dem das Selbst keinen Anteil hat. Die sogenannte 〈Selbstlosigkeit) kann auch ein Deckname dafür sein, dass die Tugend einem äußerlich geblieben ist. Deshalb kam Nietzsches Zarathustra, damit die 〈Tugendhaften〉

${ }^{41}$ AaO., 352 (Zweiter Teil).

42 Tillich 1991, 31. 
müde würden «zu sagen: «daß eine Handlung gut ist, das macht, sie ist selbstlos.> Ach, meine Freunde! Daß euer Selbst in der Handlung sei, wie die Mutter im Kinde ist: das sei mir euer Wort von Tugend!» 43 Nicht darauf kommt es an, sich selbst «selbstlos〉 los zu werden, sondern vielmehr darauf, ganz in dem präsent zu sein, was man tut. Nur wer sich selbst in seinen Taten wiederfindet, kann auch die Verantwortung für die Konsequenzen seines Tuns übernehmen.

Dazu gehört allerdings, dass sich das Selbst, das sich in etwas wiederfindet, das es nicht bejahen kann, überwindet. Im Kapitel <Von der Selbst-Überwindung» steht zu lesen: «Und dies Geheimnis redete das Leben selber zu mir: ¿Siehe〉, sprach es, ich bin das, was sich immer überwinden $m и \beta .{ }^{44}$ Das anonyme Leben wird hier personifiziert und spricht, als wäre es ein menschliches Ich, dessen Selbstbejahung Selbstverneinung einschließt, wenn nicht gar Selbstwiderspruch. Für Nietzsche ist das Leben "Kampf» und «Werden», «Zweck und der Zwecke Widerspruch ${ }^{45}$. Was das Leben auch schafft und wie es auch liebt, was es geschaffen hat, bald muss es ihm Gegner sein und sich auch gegen die eigene Liebe zum Geschaffenen wenden. So will es angeblich der «Wille» des Lebens - und dann wendet sich der Text, aus dem das Leben selbst spricht, direkt an seinen Leser: «Und auch du, Erkennender, bist nur ein Pfad und Fußtapfen meines Willens: wahrlich, mein Wille zur Macht wandelt auch auf den Füßen deines Willens zur Wahrheit!» ${ }^{46}$ Hier deutet sich ein Konflikt an zwischen der Macht des Lebens bzw. seinem Willen zur Macht und dem, was das einzelne Lebewesen mit seinem Leben will. Das eigenwillige Leben kann ihm sozusagen auf den Füßen herumtrampeln.

Dass dies nicht nur dann der Fall sein kann, wenn ein Einzelner will, was lebensverneinend ist, zeigt die Fortsetzung des Textes, in dem Zarathustra weiterredet und den «Wertschätzenden» sagt, es gebe kein «Gutes und Böses, das unvergänglich wäre» - vielmehr müsse es sich ebenfalls immer wieder überwinden: «Und wer ein Schöpfer sein muß im Guten und Bösen: wahrlich, der muß ein Vernichter erst sein und Werte zerbrechen. Also gehört das höchste Böse zur höchsten Güte: diese aber ist die schöpferische.» ${ }^{47}$ Darin liegt, dass Gut und Böse im Leben nicht feststehen. Nicht einmal die Definition des Guten als das Lebensbejahende ist haltbar, denn zum Leben gehört auch der Tod, das Lebensverneinende, und dies

\footnotetext{
${ }^{43}$ Nietzsche 1997, 353.

${ }^{44} \mathrm{AaO} ., 371$ (Zweiter Teil).

45 Ebd.

${ }^{46}$ Ebd.

$47 \mathrm{AaO}, 372$.
} 
Lebensverneinende muss auch bejaht werden, wenn das Leben als solches weitergehen soll. Damit das Leben als solches weitergehen kann, müssen die einzelnen Lebewesen sterben. Somit gehört das Böse, was dem Einzelnen den Garaus macht, zur Güte des Lebens als Gesamtheit dazu; doch sofern die Gesamtheit eine Abstraktion ist und die Wertschätzung immer nur von den Einzelnen ausgeht, sind die Werte des Einen möglicherweise im Widerstreit mit den Werten des Anderen - zumindest dann, wenn das Leben des Einen auf dem Tod des Anderen basiert. Nietzsches Überlegungen führen uns nicht nur zur «Umwertung aller Werte〉, sondern 〈jenseits von Gut und Böses. Und an dieser Stelle, wo nur noch der Kampf ums Überleben feststeht, die Werte aber ohne Rücksicht auf die Würde einzelner Lebenden ständig wechseln, wird Nietzsches Gedanke emphatischer Selbstbejahung angreifbar.

Bezeichnenderweise entging Tillich der subtile Themenwechsel im Übergang zum Kapitel 〈Von der Selbst-Überwindung〉. Hier ist nicht mehr vom einzelnen Selbst und dessen Selbstbejahung durch Selbstüberwindung die Rede, sondern vom Leben selbst, das sich bejaht, indem es sich "opfert», wie Nietzsche es nennt: «Untergang» und «Blätterfallen» gehören genauso zu ihm wie Zeugung und neue Blüte im Wechsel der Jahreszeiten. ${ }^{48}$ Was die Rede vom Sich-Opfern allerdings verdeckt, ist dies, dass es sich hierbei um einen unpersönlichen und amoralischen Vorgang handelt, der zwar natürlich ist, der aber nicht auf intra- und interpersonale Prozesse übertragen werden darf. Andernfalls besteht die Gefahr, dass Nietzsches sozialkritische Lebensphilosophie für sozialdarwinistische Zwecke missbraucht wird.

Was Tillich zwar nicht entgangen ist, ihn aber offensichtlich nicht gestört hat, ist die atheistische Ausrichtung der Sprüche Zarathustras. Das Leben hat die Rolle der höchsten Macht eingenommen - freilich einer zweideutigen Macht, die den Menschen in seinem Dasein allein lässt und sich nicht schert um dessen Freud und Leid. Angesichts dieser Macht braucht der Mensch «Einsiedlerund Adler-Mut, dem auch kein Gott mehr zusieht»: «Herz hat, wer Furcht kennt, aber Furcht zwingt; wer den Abgrund sieht, aber mit Stolz. Wer den Abgrund sieht, aber mit Adlers-Augen, - wer mit Adlers-Krallen den Abgrund faßt: Der hat Mut.» ${ }^{49}$ Tillich deutet den Abgrund als Abgrund des Nichtseins in der totalen Einsamkeit dessen, der die Botschaft annimmt, dass Gott tot ist. ${ }^{50}$ Der Mut zum Sein ist hier der Mut zum Sein ohne Gott. Wenn überhaupt noch

48 AaO., 371.

$49 \mathrm{AaO} ., 524$ (Vierter und letzter Teil).

${ }^{50} \mathrm{Vgl}$. Tillich 1991, 32. 
ein Glaube übrig geblieben ist, ist es ein Glaube an die Macht des Lebens, die Leben schafft und Leben vernichtet.

Vielleicht lässt sich Nietzsches spöttische, ironische und zynische Behandlung des Christentums als Notwehr eines hochbegabten, hypersensiblen Menschen verstehen, der unter dem gelebten Zynismus seiner (pseudo-)christlichen Umgebung litt. Er hat selbst den ¿Einsiedler-und Adler-Mut gefasst und scharfen, stolzen Blickes in den Abgrund geschaut. Vielleicht wurde er deshalb verrückt. Wie auch immer sich dies verhält, von Nietzsche haben wir bedenkenswerte Anfragen an das traditionelle Christentum geerbt. Die Frage, die bei ihm offenbleibt, ist die Frage nach einem letzten Halt - nach dem Halt, den er selbst nicht gefunden hat. Wohin sich wenden angesichts der Zwei- oder Vieldeutigkeit des Lebens, das den Tod in seinem Schoße hat? Woher die Widerstandskraft nehmen, um nicht daran zu zerbrechen? Was oder wer verdient wirklich unser Vertrauen und kann uns im Leben wie im Sterben tragen?

\section{Der Mut des Vertrauens und das Ja zum Leben}

Tillich stellt sich der Herausforderung durch Nietzsche und die Existentialisten des 20. Jahrhunderts, in deren Schriften sich der Mut der Verzweiflung manifestiert. Der Mut der Verzweiflung ist hier Selbstbejahung trotz erfahrener Sinnlosigkeit und «der Mut, aus sich zu machen, was man sein will.» ${ }^{51}$ Während in der klassischen Theologie nur Gott das Vorrecht hat, a se, aus sich zu sein, weil nichts in ihm ist, was nicht durch ihn ist, gibt der Existentialismus dem Menschen aufgrund der Feststellung, dass Gott tot ist, die göttliche Aseität. Nichts soll im Menschen sein, was nicht durch ihn ist. ${ }^{52}$ Tillich sucht eine Alternative sowohl zur klassischen Theologie wie zum Existentialismus, und zwar in Rückbesinnung auf die Reformation. Das letzte Kapitel seines Büchleins hat den Titel ‘VI. Mut und Transzendenz: Der Mut, sich zu bejahen als bejaht. Im Folgenden sei vor allem das hervorgehoben, was den Mut des Vertrauens charakterisiert, das Tillich dem Mut derVerzweiflung entgegensetzt.

Für Tillich bringt Dürers Holzschnitt 〈Ritter, Tod und Teufel〉 den Geist der lutherischen Reformation und den lutherischen Mut des Vertrauens zum Ausdruck: «ein Ritter reitet in voller Rüstung durch einen Hohlweg, von der Gestalt des Todes und der des Teufels begleitet; aber er blickt furchtlos, gesammelt und vertrauensvoll ge-

\footnotetext{
${ }^{51} \mathrm{AaO} ., 113$.

52 Vgl. aaO., 115
} 
radeaus. Er ist allein, aber nicht verlassen..$^{53}$ Aus der personhaften Begegnung mit Gott erwächst der Mut des Vertrauens auf die sich darin offenbarende personhafte Wirklichkeit. Tillich betont jedoch, dass die Identifikation des Mutes des Vertrauens mit dem Mut des Glaubens nicht berechtigt ist, da Vertrauen nur ein Element im Glauben ist: "Glaube umfaßt beides, mystische Partizipation und persönliches Vertrauen. ${ }^{54}$ Tillich zufolge transzendiert und vereint der Mut der Reformatoren beide Formen des Mutes: den Mut, Teil eines Ganzen zu sein und den Mut, man selbst zu sein. Da wir Vertrauen in Bezug auf unsere Existenz jedoch nur haben können, wenn wir unser Vertrauen nicht mehr auf uns selber gründen, kann der Mut des Vertrauens nicht im Vertrauen auf uns selber gründen. Gründet er stattdessen im Vertrauen auf Gott?

Dies ist nicht nur eine rhetorische Frage. Zunächst sieht es so aus, als folge Tillich getreu der lutherischen Tradition. Dann aber wird deutlich, dass er sich vom Theismus und Personalismus verabschiedet hat und einen sabsoluten Glauben` vertritt, der sich auch von dem Gott losgelöst hat, mit dem Luther noch per Du war. In gewissem Sinne ist diese Bewegung bei Luther selbst angelegt, der zur Erklärung des Ersten Gebots im Großen Katechismus schreibt: "Was heißt ein Gott haben oder was ist Gott? Antwort: Ein Gott heißet das, dazu man sich versehen soll alles Guten und Zuflucht haben in allen Nöten. [...] Worauf Du nu (sage ich) Dein Herz hängest und verlässest, das ist eigentlich Dein Gott. $\|^{55}$ Dies impliziert die Möglichkeit, dass man sein Herz auch an etwas anderes hängen kann als an den biblischen Gott. Wenn man sein Vertrauen auf dieses Andere setzt, fungiert dieses Andere als Gott. Allerdings wird im selben Abschnitt klar, dass das Vertrauen allein noch kein Kriterium zur Unterscheidung zwischen rechtem und falschem Glauben liefert, denn das Vertrauen kann sich sowohl auf den wahren Gott wie auch auf einen Abgott richten. Zum wahren Glauben gehört für Luther die Beziehung auf Christus, in dem sich der wahre Gott offenbart hat. Dementsprechend wendet sich seine Kritik gegen diejenigen, «qui sine Christo confidunt accedere ad Deum $»^{56}$. Gehört Tillich auch zu denen, die sich trauen, ohne Christus an Gott heranzutreten?

$53 \mathrm{AaO}, 121$.

${ }^{54} \mathrm{AaO}, 120$

${ }_{55}$ M. Luther, Der Große Katechismus, in: Bekenntnisschriften der evangelisch-lutherischen Kirche herausgegeben im Gedenkjahr der Augsburgischen Konfession 1930, hg. vom Deutschen Evangelischen Kirchenausschuss, Göttingen ${ }^{11} 1992,560$.

56 WA 56, 298, 24. Vgl. meinen Artikel «Vertrauen und/oder Gewissheit? Kontroverstheologische und religionsphilosophische Fragen im Anschluss an Luther, Kier- 
Strenggenommen tritt Tillich gar nicht an einen persönlichen Gott heran, sondern beschreibt die menschliche Seite des Glaubens, die unter allen Umständen persönlich bleibt, und zwar als eine Erfahrung, deren intentionales Objekt sich dem menschlichen Erkenntnisvermögen entzieht:

Glaube ist Ergriffensein von dem, was uns unbedingt angeht, dem Grund unseres Seins und Sinns. [...] Der Glaube ist nicht die theoretische Annahme von etwas, das erkenntnismäßig zweifelhaft ist, sondern er ist die existentielle Bejahung von etwas, das alle gegenständliche Erfahrung transzendiert. ${ }^{57}$

Dass der Grund unseres Seins und Sinns uns auch tragen kann, wird zwar nicht direkt gesagt, aber indirekt angedeutet, indem dessen Auswirkung auf den Menschen beschrieben wird. Der Mensch wird ergriffen «von der Macht des Seins trotz der überwältigenden Erfahrung des Nichtseins», und dieses Ergriffensein ist ein Glaube, «der den Mut derVerzweiflung möglich macht», d.h. den Mut,Zweifel und Sinnlosigkeit in sich hineinzunehmen. ${ }^{58}$ Dieser Glaube hat keinen spezifischen Inhalt mehr. Er ist undefinierbar: absoluter Glaube. Strukturell besteht er aus drei Elementen: erstens aus der Vitalität, die sich eines verborgenen Sinnes selbst in der Zerstörung bewusst ist; zweitens aus der Abhängigkeit der Erfahrung des Nichtseins von der Erfahrung des Seins, und drittens aus der Erfahrung des Bejahtseins. ${ }^{59}$ Aber wie denn kann die anonyme Seinsmacht eine Erfahrung des Bejahtseins hervorrufen?

Diese Frage findet eine überraschende Antwort: Einzig die Kirche unter dem Kreuz könne sich zu dem Gott über dem Gott des Theismus erheben und den Mut der Verzweiflung vermitteln "die Kirche, die den Gekreuzigten predigt, der den Gott anrief, der Gott blieb, nachdem der Gott des Vertrauens ihn in dem Dunkel der Verzweiflung und der Sinnlosigkeit verlassen hatte» ${ }^{60}$. Tillich versucht also gerade nicht, sich Gott ohne Christus zu nähern. Doch schwindet ihm im Blick auf die Gottverlassenheit des Gekreuzigten der Mut des Vertrauens, der 〈Trotzdem〉 sagt selbst zum Tode. Wie aber kann dann noch ein Ja zum Leben gesprochen werden? Oder anders gefragt: Was bedeutet dann der «Mut zum Sein`?

kegaard und Wittgenstein" (erscheint in dem von I.U. Dalferth und S. Peng-Keller herausgegebenen Tagungsband Glaube, Hoffnung, Vertrauen. Fiducia als Quaestio disputata). 57 Tillich 1991, 128.

$58 \mathrm{AaO}, 130$.

${ }^{59} \mathrm{Vgl}$. aaO., 131.

${ }^{60} \mathrm{AaO}, 138$ 
Der Mut zum Sein ist laut Tillich «sowohl der Mut derVerzweiflung wie der Mut in allem Mut und über allem Mut» ${ }^{61}$. Er sei ohne die Sicherheit, die Worte und Begriffe vermitteln, ohne Namen, ohne Kirche, ohne Kult, ohne Theologie. Aber er sei in der Tiefe von ihnen allen wirksam. Er sei die Macht des Seins, an dem sie alle partizipieren und dessen fragmentarische Ausdrucksformen sie sind. «Der Mut zum Sein gründet in dem Gott, der erscheint, wenn Gott in der Angst des Zweifels untergegangen ist. $\rangle^{62}$ Mit diesem Satz beendet Tillich seine kleine Schrift. Er nähert sich in ihr nicht dem Gott, den Jesus vertrauensvoll als seinen Vater angerufen hat, sondern dem 〈Gott über Gott» - ohne ihm zu nahe treten zu können. Denn eine Annäherung an ihn gibt es allenfalls dadurch, dass man am Leben bleibt und den Mut nicht aufgibt.

Aber, so sei abschließend gefragt, ist es möglich, den Mut des Vertrauens aufzugeben, ohne zu verzweifeln? Aus der Verzweiflung folgt ja nicht notwendigerweise der Mut derVerzweiflung, und der Mut der Verzweiflung ist nicht notwendigerweise der Mut zum Sein. Im schlimmsten Falle führt die Verzweiflung dazu, dass das Sein vom Nichtsein, das Leben vom Tode verschluckt wird. Soviel dürften die Schlüsselszenen des Kampfes um Leben oder Tod gezeigt haben. Zwar muss auch die Selbstbejahung des Vertrauens durch die Selbstverneinung der Verzweiflung hindurch, doch gibt es kein Zurück, wenn wir bei Letzterer stehenbleiben. Dann ist die Krankheit zum Tode unheilbar und führt in den Tod hinein, aber nicht mehr aus ihm heraus. Dass damit nicht zu spaßen ist, auch wenn es sich (nur) um den Tod im Leben handelt, haben wir von Kierkegaards Nosologie gelernt. Der Tod mitten im Leben ist vielleicht sogar der qualvollste und längste. Deswegen hat, wer sich verloren hat und sich im eigenen Leben nicht mehr orientieren kann, noch nicht alles verloren, sondern immer noch mehr zu verlieren, sollte es nicht glücken, sich wieder zu gewinnen. Ein Leben, das man gleichsam «ohne sich〉 lebt, verdient diesen Namen nicht mehr. Lebenswert ist es nur dann, wenn man 〈sich selbst dabei hat, denn nur dann bleibt man offen für Unvorhersehbares und kann das entgegennehmen, was dem eigenen Leben eine gute Wendung gibt.

Was einen öffnet oder offenhält, ist nichts anderes als selbstverwandelndes Vertrauen, und nur das Vertrauen auf einen Anderen kann einen über sich selbst hinaus und über verzweifeltes Selbstsein

$61 \mathrm{AaO} ., 139$

62 Ebd. 
hinweg führen. ${ }^{63}$ Zum Schluss sei noch aus dem Brief eines Denkers zitiert, der selbst kurz vor dem Selbstmord stand, aber gerade noch von etwas in ihm zurückgehalten wurde. In seinem Brief an Gertrud Oppenheim vom 31. Mai 1917 weist Franz Rosenzweig darauf hin, dass der kategorische Imperativ dem Einzelnen zwar klar ist, die Gestalt des Endzustands wie auch der Weg dahin der Einsicht der Vernunft jedoch entzogen ist. Zwar könne und müsse man die Weltgeschichte deuten, die Deutung bleibe aber immer hypothetisch und voraussagen lasse sich gar nichts: «es steht nichts in unserer Macht als der jeweils unmittelbar nächste Schritt, die «Forderung des Tages`; nur ihr gilt das «du kannst, denn du sollst». Schon für den morgigen Tag braucht man die ganze Kraft des Vertrauens, um nicht zu verzweifeln.» ${ }^{64}$ Ohne Vertrauen können wir nicht leben. Ob das, worauf wir unser Vertrauen setzen, uns auch wirklich einen letzten Halt gibt, wissen wir nicht im Voraus. Doch ohne Vertrauen werden wir es auch nie erfahren.

- Dr. Claudia Welz ist Associate Professor am Center for Subjectivity Research der Universität Kopenhagen. Ihre Habilitation trägt den Titel «Vertrauen und Versuchung».

\footnotetext{
${ }^{63}$ Dies habe ich an anderer Stelle näher ausgeführt, vgl. die Artikel Trust as Basic Openness and Self-Transcendence, in: Trust, Sociality, Selfhood (Religion in Philosophy and Theology 52), hg. von A. Grøn/C. Welz, Tübingen 2010, 45-64, und Selbstwerdung im Angesicht des Anderen: Vertrauen und Selbstverwandlung bei Kierkegaard und Rosenzweig) (erscheint in einem von H.M. Dober herausgegebenen Band der Rosenzweigiana).

${ }^{64}$ F. Rosenzweig, Der Mensch und sein Werk. Gesammelte Schriften, Bd. 1: Briefe und Tagebücher 1900-1918, hg. von R. Rosenzweig/E. Rosenzweig-Scheinmann, Den Haag 1979, 415
} 